\title{
Repeated Episodes of Umbilical Cord Occlusion in Fetal Sheep Lead to Preferential Damage to the Striatum and Sensitize the Heart to Further Insults
}

\author{
E. C. MALLARD, C. E. WILLIAMS, B. M. JOHNSTON, M. I. GUNNING, S. DAVIS, AND \\ P. D. GLUCKMAN \\ Research Centre for Developmental Medicine and Biology, University of Auckland, Auckland, \\ New Zealand
}

\begin{abstract}
ABST
The effect of repeated episodes of asphyxia on the fetal
cardiovascular system and CNS was examined. The umbilical cord was occluded for 5 min, four times, at 30 -min intervals in 11 chronically instrumented fetal sheep (118-126 d). Fetal electrocorticogram (ECoG), cortical impedance, ECG, heart rate, and blood pressure were continuously recorded for $3 \mathrm{~d}$, after which neuronal loss was determined histologically. Each occlusion resulted in fetal hypoxemia and bradycardia accompanied by increased T/QRS ratio. Progressively severe hypotension and lactic acidosis developed during successive occlusions. The ECoG was depressed and cortical impedance increased with each occlusion. During the final occlusion, blood pressure fell to $3.5 \pm$ $1 \mathrm{kPa}$ and heart rate to $93 \pm 9 \mathrm{bpm}$, T/QRS ratio increased to $0.44 \pm 0.3$, and lactate rose to $7.2 \pm 1.2 \mathrm{mM} / \mathrm{L}$. Three animals died from cardiac fibrillation during recirculation after the third or fourth occlusion. After the asphyxial episodes, blood pressure and heart rate returned to normal, and the $\mathrm{T}$ wave was inverted for $310 \pm 155 \mathrm{~min}$. Lactate returned to baseline within $24 \mathrm{~h}$. The ECoG remained depressed for $90 \pm 35 \mathrm{~min}$, and intermittent
\end{abstract}

seizures developed at $3.3 \pm 1.4 \mathrm{~h}$ after the last occlusion. Neuronal loss was primarily found in the striatum. The extent of neuronal loss correlated with the degree of hypotension, increase in T/QRS ratio, duration of postasphyxial ECoG depression, and number of seizures. These results indicate that transient asphyxial episodes compromise the ability of the heart to tolerate additional insults and further suggest that neuronal loss is a consequence of cardiovascular compromise secondary to asphyxia. Therefore, repetitive asphyxial episodes may lead to intrauterine death or striatal damage in survivors. (Pediatr Res 37: 707-713, 1995)

\section{ECoG, electrocorticogram}

Abbreviations

CI, cortical impedance

HR, heart rate

MAP, mean arterial blood pressure

GABA, $\gamma$-amino butyric acid
Prenatal asphyxia is thought to be a cause of intrauterine death or impaired postnatal neurologic development in survivors. However, specific cause and effect relationships have been difficult to establish in the human fetus because of inadequate means of monitoring fetal events. We have previously shown that repeated episodes of cerebral ischemia in the fetal sheep alter the degree and distribution of brain injury compared with single insults (1). In particular, these repeated insults result in the loss of striatal neurons.

Intermittent partial umbilical cord occlusion has been reported to cause cerebral white matter injury in fetal sheep (2). Others

Received July 12, 1994; accepted December 20, 1994

Correspondence and reprint requests: E. C. Mallard, Research Centre for Developmental Medicine and Biology, Department of Paediatrics, University of Auckland, Private Bag 92019, Auckland, New Zealand.

Supported by a program grant from the Health Research Council of New Zealand and a grant from the New Zealand Lottery Grants Board. have shown increasing acidosis and electrocardiographic evidence of asphyxia during repeated umbilical cord occlusions (3). However, the relationship between cardiac function, cerebral function, and neuronal loss after recurrent episodes of fetal asphyxia is not clear. We have previously shown that systemic asphyxia induced by umbilical cord occlusion in fetal sheep results in hippocampal injury, the severity of which is related to the degree of associated arterial hypotension (4). It is likely that, when the human fetus is compromised, multiple episodes of asphyxia occur rather than a single prolonged event. Interactions between asphyxial episodes may be an important determinant of both the extent and the localization of neuronal loss.

The purpose of the present study was to examine cerebral and cardiac function during and after repeated umbilical cord occlusions in the sheep fetus and to determine their relationship to neuropathologic outcome. 


\section{METHODS}

Surgical protocol. These studies were approved by the Animal Ethical Committee of the University of Auckland. Eleven fetal sheep (118-126 d of gestation) were operated on under halothane anesthesia $(2 \%)$ with sterile techniques as previously described (4). Briefly, polyvinyl catheters were inserted into the brachial arteries and one catheter was placed in the amniotic cavity. A reversible inflatable umbilical cuff (In Vivo Metric, Healdsburg, CA) was placed around the umbilical cord. Two pairs of ECoG electrodes (Cooner wire AS6335SSF; Cooner Wire Co., Chatsworth, CA) were placed on the parietal parasagittal dura through burr holes (skull coordinates relative to bregma: anterior $5 \mathrm{~mm}$ and $15 \mathrm{~mm}$, lateral of midline $10 \mathrm{~mm}$ ). To record $\mathrm{CI}$, two stimulating electrodes (Cooner wire AS633-3SSF) were placed $10 \mathrm{~mm}$ anterior of bregma and $15 \mathrm{~mm}$ lateral of midline. One pair of ECG electrodes (Cooner wire AS633-3SSF) were placed s.c., one over the apex of the heart and one on the opposite shoulder. The maternal femoral vein was catheterized. After the operation, the ewe was housed with free access to water and hay supplemented with sheep nuts and alfalfa. Antibiotics (penicillin to the ewe and gentamicin to the fetus) were administered for $3 \mathrm{~d}$ after surgery and on the day of study.

Experimental protocol. Experiments were performed only in healthy fetuses $\left(\mathrm{PO}_{2}>2.5 \mathrm{kPa}\right.$, lactate $\left.<1.1 \mathrm{mmol} / \mathrm{L}\right)$ and were commenced 3 to $5 \mathrm{~d}$ after surgery. Fetal ECoG, CI, MAP, and HR were recorded continuously for $12 \mathrm{~h}$ before the first occlusion and for $72 \mathrm{~h}$ after the last occlusion. ECG was recorded continuously for $1 \mathrm{~h}$ before the first occlusion and for $10 \mathrm{~h}$ after the last occlusion. After collection of baseline data, the umbilical cord was transiently occluded for $5 \mathrm{~min}$ and then released. This procedure was repeated four times, separated by 30-min intervals. Each occlusion resulted in an isoelectric ECoG (amplitude $<5 \mu \mathrm{V}$ ), as displayed on the chart recorder. Fetal arterial blood gases were sampled at the following time points: $30 \mathrm{~min}$ before the first occlusion; immediately before and at the end of each occlusion; and at 30,60, 120, 180, 240 $\min$ and 24,48 , and $72 \mathrm{~h}$ after the last occlusion. Three days after the last occlusion, sheep were killed by an overdose of pentobarbitone $(3.5 \mathrm{~g})$, and the fetal brain was prepared for histologic analysis as previously described (5). Briefly, the brain was perfused in situ with $4 \%$ paraformaldehyde in $0.2 \mathrm{M}$ phosphate buffer, paraffin-embedded, and coronally sectioned at 8- $\mu \mathrm{m}$ thickness. Histologic sections were stained with thionine and acid fuchsin. This procedure stains dead acidophilic neurons pink (6).

Recordings. The ECoG signal was low-pass filtered before sampling, and the intensity spectrum and the CI signal were extracted from the ECoG as previously described (7). CI increases concomitantly with the development of cytotoxic edema (7). MAP was recorded from the brachial artery (Novatrans II, MX860, Medex Inc., Hilliard, OH) and adjusted for amniotic pressure. HR was derived from the brachial arterial pressure. ECoG intensity, CI, MAP, and HR were processed on-line, averaged over 1-min intervals, and each block of data was stored to a disk (Labview for Windows, National Instruments, Austin, TX). An additional channel of raw ECoG was recorded for $30 \mathrm{~min}$ before the first occlusion and for $36 \mathrm{~h}$ after the last occlusion and stored to a disk for off-line analysis. ECG was filtered by a first-order high-pass filter $(0.1 \mathrm{~Hz})$ and an eighth order low-pass Bessel filter $(80 \mathrm{~Hz})$ before recording. During the experiments, signals were displayed on the computer monitor and on an analog chart recorder running at 5 $\mathrm{mm} / \mathrm{min}$.

Analysis. Off-line analysis was performed using Viewdac signal analysis program (Keithley Instruments, Inc., Taunton, MA). The total ECoG intensity was derived from 1 to $30 \mathrm{~Hz}$, $\log$ transformed and normalized with respect to the $12 \mathrm{~h}$ baseline as previously described (5). CI was normalised and expressed as a percentage of baseline. Total ECoG intensity, ECoG spectral edge (lower 90\% of frequency) and CI were measured during each insult and in five hour bins after the last occlusion. The ECoG depression following the last occlusion was defined as intensity reduced by more than $5 \mathrm{~dB}$ from preinsult. Seizure and spike detection software (Monitor, Stellate Systems, Quebec, Canada) was used to identify episodes of electrical seizure activity and individual spikes. Number of seizures and spike transients were determined. In addition, a pediatric neurologist (S.D.) visually examined each raw ECoG record for seizure activity, defined as paroxysmal high-voltage $(>1 \mathrm{mV})$ discharges of $>30 \mathrm{~s}$ duration. For automatic detection, seizures and spikes were defined according to the default settings of Monitor software except for the maximum coeffcient of variation for seizures, which was set at 60 , and the spike recognition amplitude threshold, which was set at $8(8$, 9).

ECG complexes were digitally high-pass filtered (finite pulse response, $1.5 \mathrm{~Hz}$ ) and averaged over 1-min intervals with respect to the $S$ wave peak. Baseline was defined as the $30-\mathrm{ms}$ point before the $S$ peak (Fig. 1). The amplitude of the QRS complex was calculated as the distance between the baseline and the peak of the S wave. The ST segment was defined as the

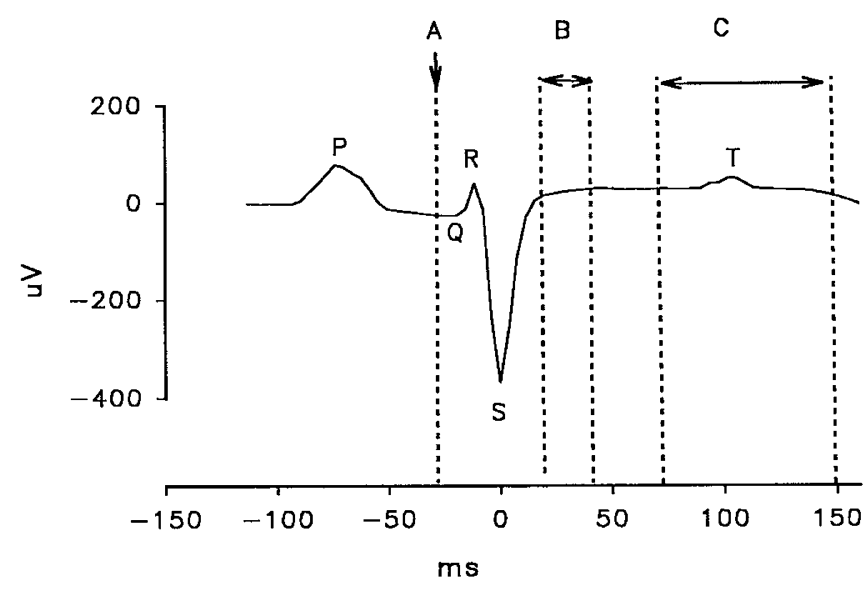

Figure 1. Diagram illustrating measurements of $S T / Q R S$ ratio and T/QRS ratio. The baseline $(A)$ was defined $30 \mathrm{~ms}$ before the $S$ wave peak. The amplitude of the QRS complex was measured between the baseline and the peak of the S wave. The ST segment $(B)$ was defined as the interval between 20 and $40 \mathrm{~ms}$ after the $S$ wave peak. The $T$ wave $(C)$ was defined as the interval 75-150 ms after the $S$ wave peak. The ST/QRS ratio was calculated as the ratio between the amplitude of the ST segment and the QRS complex. The ratio between the amplitude of the $\mathrm{T}$ wave and the QRS was calculated as the T/QRS ratio. 

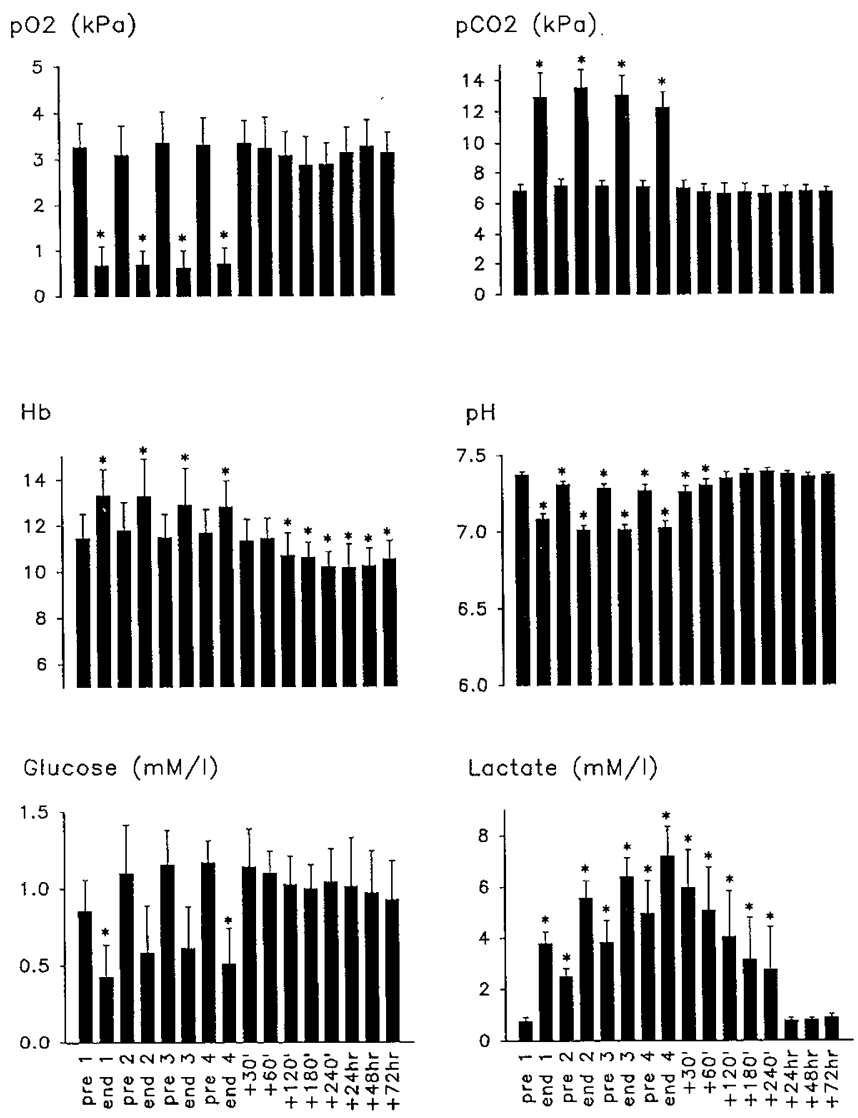

Figure 2. Fetal arterial blood gas and metabolic measurements during and after asphyxia. Lactate levels progressively increased with successive occlusions and were elevated by $240 \mathrm{~min}$ after the last occlusion but had returned to baseline by $24 \mathrm{~h} .{ }^{*}, p<0.05$ compared with baseline.

interval $20-40 \mathrm{~ms}$ after the $\mathrm{S}$ wave peak, and the $\mathrm{T}$ wave was defined as the interval 75-150 ms after the S peak (Fig. 1). Changes in the ST segment were determined as the ratio between the amplitude of the ST segment and the QRS segment (ST/QRS). The T/QRS ratio was calculated as the ratio between the amplitude of the $\mathrm{T}$ wave and the amplitude of the QRS complex. Duration of postasphyxial depression of the $\mathrm{ST} / \mathrm{QRS}$ ratio and T/QRS ratio was calculated on smoothed $(0.01-\mathrm{Hz})$ time series.

We have previously shown that umbilical cord occlusion in fetal sheep causes bilateral cerebral damage (4). Therefore, the left hemisphere is representative of damage in the whole brain and was used for histologic analysis. The numbers (in $\mathrm{mm}$ ) in parentheses refer to cerebral regions according to the stereotaxic atlas of the fetal sheep (10). Examination for neuronal loss was performed in the following regions: striatum $(29 \mathrm{~mm})$, thalamus $(20 \mathrm{~mm})$, parasagittal cortex $(29 \mathrm{~mm}$ and $20 \mathrm{~mm})$, and dorsal and ventral hippocampus, including dentate gyrus and CA1-4 (13 $\mathrm{mm}$ and $15 \mathrm{~mm})$. Acidophilic neurons with pyknotic nuclei were defined as dead neurons (5). Each brain region was analyzed according to the following scale: $0=$ no damage, $1=<20 \%, 2=20-70 \%$, and $3=>70 \%$ neuronal loss. The striatum was given two scores, one for the caudate nuclei and one for the putamen. The thalamus was given one score. Parasagittal cortex was given two scores, one at anterior $(29 \mathrm{~mm})$ and one at posterior $(20 \mathrm{~mm})$ level. The hippocampus
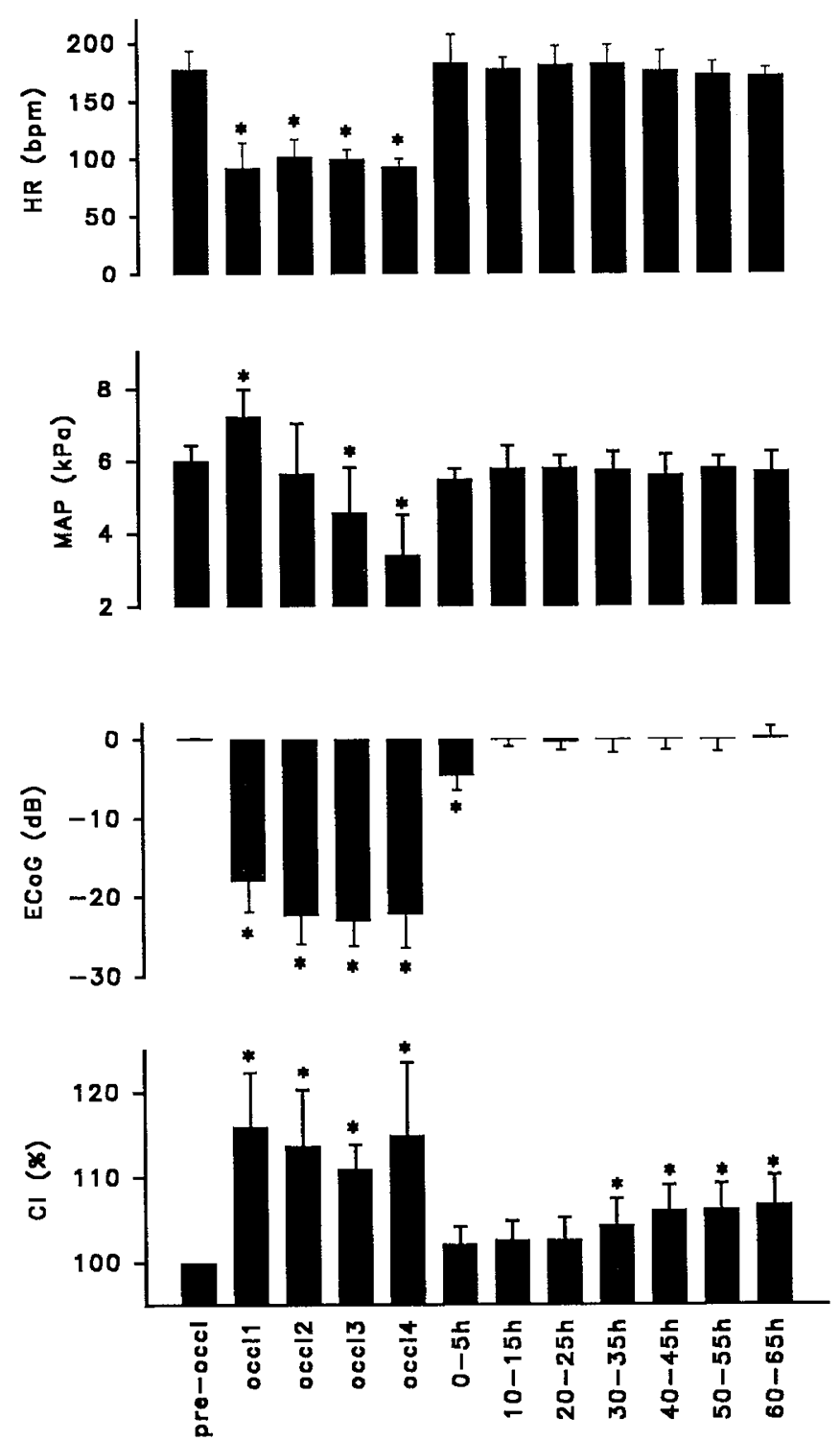

Figure 3. HR, MAP, ECoG, and $\mathrm{CI}$ during and after repeated umbilical cord occlusion. Arterial blood pressure became increasingly hypotensive with each occlusion. ${ }^{*}, p<0.05$ compared with baseline.

was given two scores, one at $15 \mathrm{~mm}$ and one at $13 \mathrm{~mm}$. Average scores were calculated for each region. The scores within each region were added to give each animal a total neuronal loss score.

Statistical analysis of physiologic parameters was performed by analysis of variance, with time as repeated measure. Histologic neuronal loss scores were compared using KruskalWallis analysis of variance on ranks. When overall differences were found, specific changes were determined by Newman Keul's multiple comparison test. Correlations were performed on rank transformed data. Data are presented as mean $\pm \mathrm{SD}$.

\section{RESULTS}

Three animals died on recirculation from occlusion due to cardiac fibrillation, two immediately after the third occlusion and one after the fourth occlusion. These animals were excluded from analysis. 

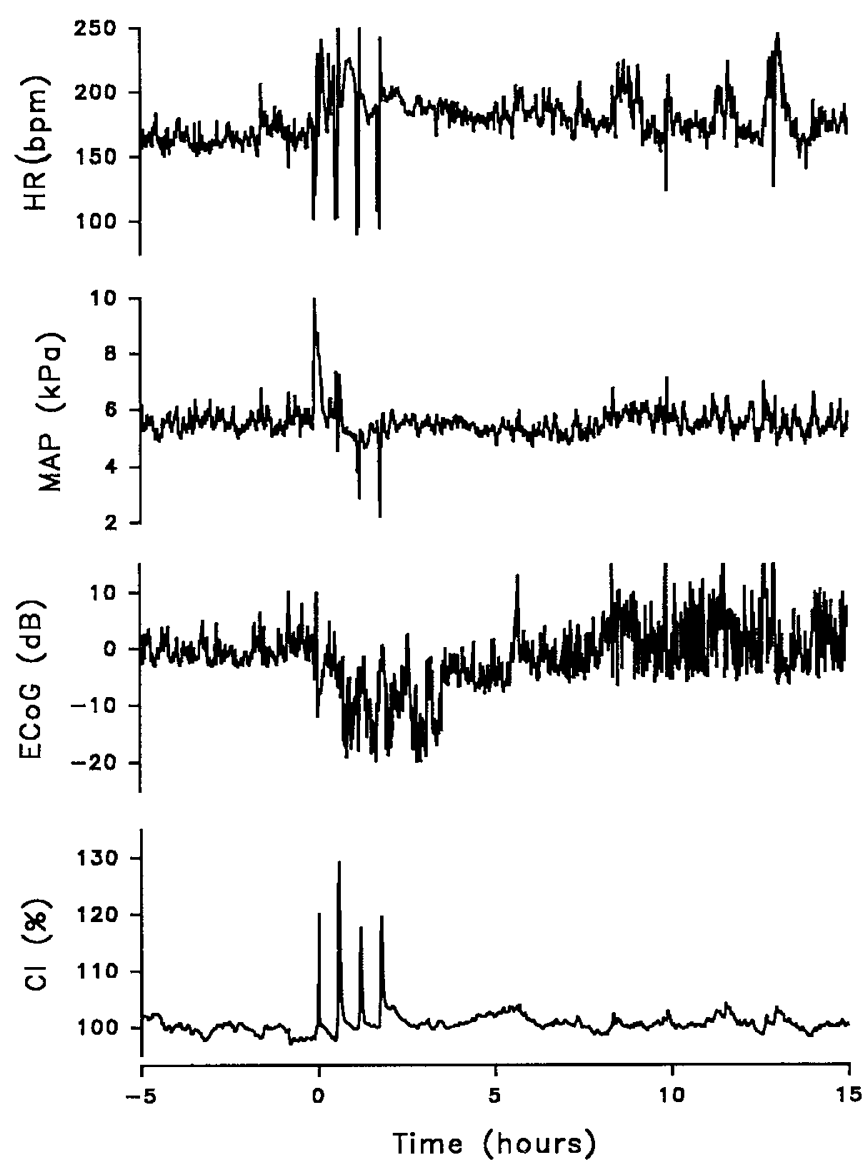

Figure 4. Typical example of HR, MAP, ECoG, and CI during and after repeated umbilical cord occlusions. Bradycardia developed with each occlusion and the arterial blood pressure became progressively hypotensive with successive insults. ECoG became severely depressed during occlusion and remained depressed between occlusions. A similar increase in CI was observed with each insult.

The first cord occlusion resulted in transient severe asphyxia, including hypoxemia $\left(\mathrm{Po}_{2} 0.7 \pm 0.4 \mathrm{kPa}\right)$, hypercarbia $\left(\mathrm{PCO}_{2} 12.9 \pm 1.5 \mathrm{kPa}\right)$, and acidosis (lactate $3.81 \pm 0.4 \mathrm{mM} / \mathrm{L}$, pH $7.08 \pm 0.02$ ) (Fig. 2). The majority of blood gas and metabolic measurements showed similar changes with each successive occlusion. The exception was arterial lactate, which showed a progressive increase in lactate with each occlusion and after each recirculation lactate remained high. After the last occlusion, arterial lactate was increased for at least 240 min, but had returned to baseline by $24 \mathrm{~h}$.

MAP showed variable changes during the occlusions (Figs. 3 and 4). The first occlusion resulted in arterial hypertension throughout the occlusion, after which there was a progressive hypotension at the end of each occlusion. Postasphyxial MAP was normal. Bradycardia developed during each occlusion of similar severity (Figs. 3 and 4), and upon recirculation HR returned to normal. There were no secondary changes in HR after the insults.

ECG wave form analysis revealed large positive and negative deviations in the ST segment during the occlusions with no consistent pattern. After the last occlusion, the ST segment was depressed for $270 \pm 110 \mathrm{~min}$. The T/QRS ratio increased during each occlusion (Fig. 5). After the last occlusion, the T

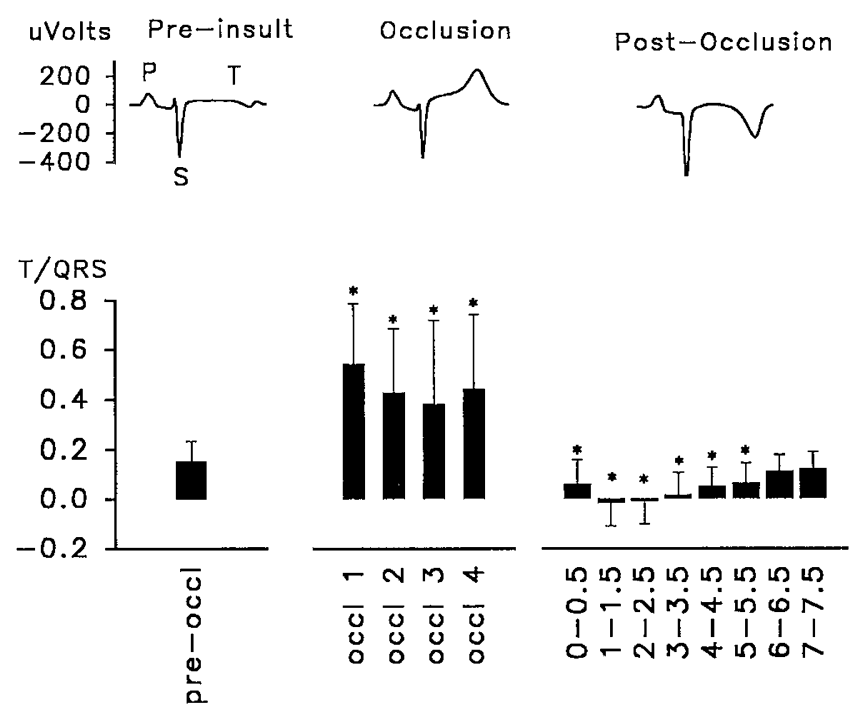

Figure 5. Changes in the T/QRS ratio during and after repeated umbilical cord occlusion. Upper panel shows a typical example of the electrocardiographic changes. Each occlusion results in an increase in the T/QRS ratio. After the last occlusion, the T/QRS ratio decreased below preinsult values and the $\mathrm{T}$ wave became inverted. ${ }^{*}, p<0.05$ compared with baseline.

wave became inverted and the T/QRS ratio decreased below preinsult values for $310 \pm 155 \mathrm{~min}$.

Each occlusion resulted in severely reduced ECoG intensity ( $p<0.05$, Figs. 3 and 4 ). Depression of ECoG intensity was more severe during the second, third, and fourth occlusion compared with the first insult $(p<0.05)$. There was no difference in intensity between the last three occlusions. After the fourth occlusion, ECoG intensity was suppressed for an additional $90 \pm 35 \mathrm{~min}$ then recovered to preinsult levels. During occlusions, spectral edge ECoG fell from preinsult levels $(13.2 \pm 1.6 \mathrm{~Hz})$ to $9.1 \pm 1.9 \mathrm{~Hz}$. After the last insult, the ECoG spectral edge frequency recovered toward preinsult levels and was not different from baseline at $120 \mathrm{~min}$ after the last occlusion. Spectral edge frequency remained unchanged throughout the rest of the experiment.

One animal was excluded from the seizure detection and two animals were excluded from spike detection analysis because of technical difficulties. Seizures occurred in five of seven animals, starting at $3.3 \pm 1.4 \mathrm{~h}(2.0-5.6 \mathrm{~h})$ after the last umbilical cord occlusion, with most seizures occurring between 11 and $15 \mathrm{~h}$ (Fig. 6). All animals demonstrated spike activity. The amplitudes of the spike waves were $18-450 \mu \mathrm{V}$, starting immediately after the first occlusion and with the highest rate at 11-15 h after the last occlusion (Fig. 6).

$\mathrm{Cl}$ increased during each occlusion (Figs. 3 and 4). Each occlusion resulted in a similar increase. After each recirculation, CI returned toward preinsult values. At $30-35 \mathrm{~h}$ postinsult there was a small increase $(104 \pm 3 \%)$ in Cl compared with preinsult that persisted until the end of the experiment ( $p$ $<0.05$ ).

Histologic neuronal loss in the striatum $(1.9 \pm 0.8)$ was found in all eight surviving animals (Fig. 7). Dead neurons appeared shrunken with pyknotic nuclei and pink cytoplasm. Three animals showed severe injury throughout the striatum. In animals with mild striatal damage, the neuronal loss was in the 

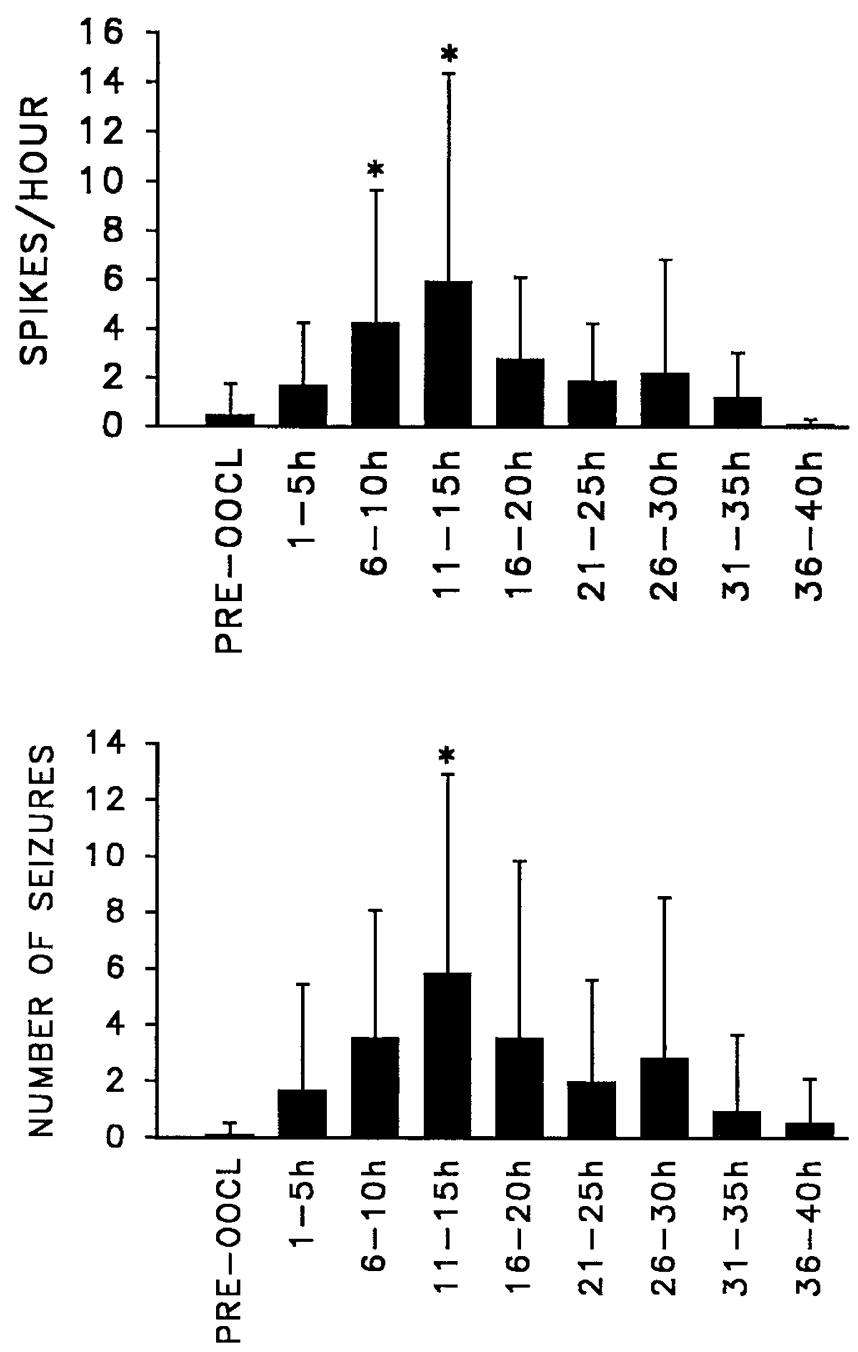

Figure 6. Number of spikes per hour and number of seizures after repeated umbilical cord occlusion. The highest number of seizures and spikes occurred $11-15 \mathrm{~h}$ after the last occlusion. ${ }^{*}, p<0.05$ compared with preinsult.

putamen. Mild-to-moderate neuronal loss was observed in the globus pallidus $(1.1 \pm 1.0)$. Mild injury was found in the hippocampus $(0.4 \pm 0.7)$ and cortex $(0.3 \pm 0.4)$. No neuronal loss was found in the thalamus.

The degree of MAP reduction and the amplitude of the T/QRS ratio during the last occlusion were positively correlated with the extent of total neuronal loss (Table 1). There was no correlation between the extent of total neuronal loss and peak arterial lactate, glucose, $\mathrm{Po}_{2}, \mathrm{pH}$, and the degree of bradycardia (Table 1). Postinsult, the duration of ECoG depression and the number of seizures were correlated with the degree of total neuronal loss (Table 1).

\section{DISCUSSION}

The present study shows that four repeated episodes of asphyxia, each of $5 \mathrm{~min}$ duration, in the late gestation fetal sheep result in neuronal loss predominantly within the striatum. In contrast, an isolated 10-min episode of umbilical cord occlusion in fetal sheep results in hippocampal damage (4). A similar change in distribution of injury was seen after repeated cerebral ischemia insults in fetal sheep. Whereas a single

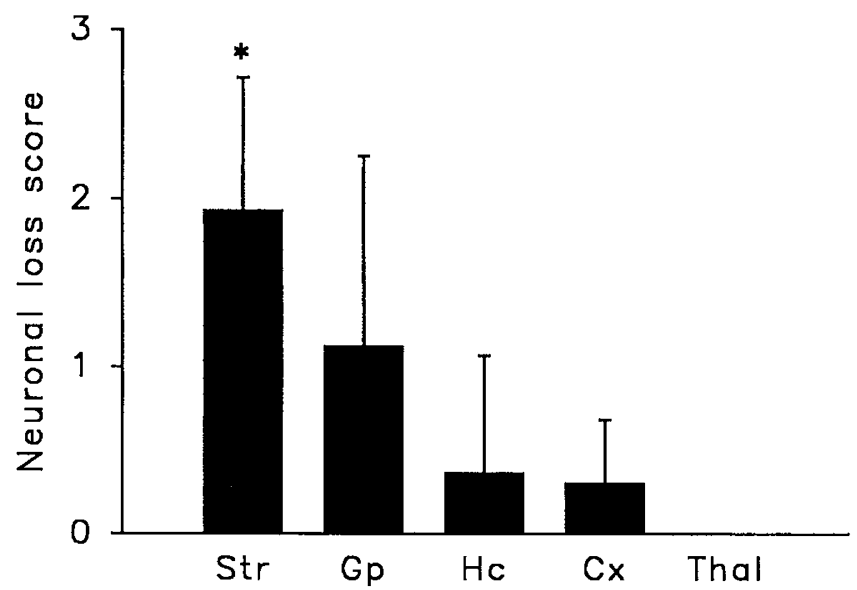

Figure 7. Histologic neuronal loss after repeated umbilical cord occlusion. The striatum showed significantly more damage than any other region. Scores: $0=$ no neuronal loss, $1=<20 \%$ neuronal loss, $2=20-70 \%$ neuronal loss, and $3=>70 \%$ neuronal loss. Str, striatum; Gp, globus pallidus; $H c$, hippocampus; $C x$, parasagittal cortex; and Thal, thalamus. ${ }^{*}, p<0.05$ compared with any other region.

episode of cerebral ischemia causes cortical and hippocampal injury, repeated brief episodes of cerebral ischemia sensitize the striatum to damage $(1,5)$. Thus it seems that preferential striatal injury is dependent on the temporal sequencing of the asphyxial/ischemic insult and not the nature of the insult.

Fetal arterial blood gas measurements showed evidence of severe asphyxia during each occlusion. Only arterial lactate demonstrated a cumulative effect with each occlusion. However, only modest increases in arterial lactate levels were reached, and there was no relationship between peak lactate level and neuronal loss. The role of elevated lactate levels in perinatal brain injury remains a controversial issue (11). In the present study, moderate lactic acidemia does not seem to be related to neuronal outcome.

There was a progressive fall in arterial blood pressure with each cord occlusion, suggesting a cumulative effect on cardiovascular function. In agreement with previous studies, we found that the degree of hypotension, but not hypoxemia, during the last umbilical cord occlusion was correlated with poor neuronal outcome $(4,12)$. During moderate asphyxia, arterial blood pressure and peripheral vascular resistance are increased and blood flow to vital organs such as heart, brain, and adrenals is increased (13). However, during severe asphyxia, peripheral vascular resistance may decrease and circulatory decentralization may occur, resulting in a reduction in blood flow to the heart and brain (14). Hypotension during asphyxia may fall below the lower limit of autoregulation in the fetus and therefore cause a decrease in cerebral blood flow (15). Hence, in the present study, it is likely to be hypotension combined with hypoxemia, secondary to asphyxia, which results in cerebral hypoxia-ischemia. Thus, it is to be anticipated that in this study it is the degree and duration of hypotension that is predictive of outcome.

Although severe bradycardia developed during each occlusion, there was not an additive effect with repeated insults and there was no correlation of the severity of bradycardia and outcome. During bradycardia, there were variable changes in the ECG wave forms. 
Table 1. Correlations between total neuronal loss score and changes in HR, MAP, T/QRS, arterial lactate, glucose, and PO $\mathrm{O}_{2}$ during the fourth umbilical cord occlusion or between total neuronal loss and duration of EEG depression and number of seizures after the fourth occlusion

\begin{tabular}{|c|c|c|c|c|c|c|c|c|c|}
\hline & HR (\% of baseline) & MAP (\% of baseline) & T/QRS & $\mathrm{PO}_{2}(\mathrm{kPa})$ & $\mathrm{pH}$ & $\begin{array}{l}\text { Lactate } \\
(\mathrm{mM} / \mathrm{L})\end{array}$ & $\begin{array}{l}\text { Glucose } \\
(\mathrm{mM} / \mathrm{L})\end{array}$ & $\begin{array}{c}\text { EEG } \\
\text { depression } \\
(\mathrm{min})\end{array}$ & $\begin{array}{c}\text { Number of } \\
\text { seizures }\end{array}$ \\
\hline Neuronal loss & $\begin{array}{l}r=0.28 \\
p=0.54\end{array}$ & $\begin{array}{l}r=0.867 \\
p=0.01\end{array}$ & $\begin{array}{l}r=0.75 \mathrm{p} \\
p=0.03\end{array}$ & $\begin{array}{l}r=0.50 \\
p=0.26\end{array}$ & $\begin{array}{l}r=-0.71 \\
p=0.08\end{array}$ & $\begin{array}{l}r=0.49 \\
p=0.26\end{array}$ & $\begin{array}{l}r=0.13 \\
p=0.78\end{array}$ & $\begin{array}{l}r=0.78 \\
p=0.02\end{array}$ & $\begin{array}{l}r=0.95 \\
p<0.01\end{array}$ \\
\hline
\end{tabular}

Several previous clinical and experimental studies have observed increases in the $\mathrm{T}$ wave during acute fetal hypoxemia and asphyxia $(16,17)$. These changes have been attributed to anaerobic myocardial metabolism (18). After repeated umbilical cord occlusion in fetal sheep, an increase in T/QRS ratio correlated with fetal acidemia and duration of elevated blood pressure during occlusions (3). In the present study, we found a correlation between the severity of neuronal loss and both the degree of hypotension and the increase in $\mathrm{T} / \mathrm{QRS}$ ratio. These correlations may suggest a functional relationship between compromised cardiac function and cerebral injury.

Three animals died due to ventricular fibrillation on reperfusion after the third and fourth episodes of umbilical cord occlusion. Repeated myocardial ischemia cause impaired recovery of cardiac function in dogs (19). Reperfusion injury due to the formation of oxygen free radicals can occur after transient myocardial ischemia (20). Free radical activity may cause ventricular fibrillation. Repeated reperfusion in the present study may have caused cumulative damage, resulting in ventricular fibrillation and fetal death.

Electrocortical activity, as indicated by ECoG intensity function, was severely depressed during and between the periods of umbilical cord occlusion, but recovered within $90 \pm$ 35 min after the last occlusion. In the present study, duration of recovery of ECoG intensity was correlated with the degree of total neuronal loss. A previous study found that prolonged ( $>4$ h) postinsult ECoG depression was associated with severe cortical neuronal loss after cerebral ischemia in fetal sheep (5). The more rapid recovery of ECoG intensity in this study is compatible with the observation of no or minimal cortical damage.

Continuous cortical seizure activity after $30 \mathrm{~min}$ of cerebral ischemia in fetal sheep is associated with infarction of the underlying cortex (5). In contrast, intermittent seizures develop when mild-to-moderate degrees of cortical injury are observed after uterine artery occlusion in fetal sheep (12). In the majority of animals, we found episodes of spike discharges and intermittent seizure activity, superimposed on normal activity, after repeated episodes of systemic asphyxia. The number of seizures was strongly related to the degree of total neuronal loss. Only minimal cortical injury was observed in four animals in the present study, while deeper structures such as the striatum were frequently severely damaged. Thus, the mechanisms of the initiation of the seizure activity seen in the present study may be different compared with the previous fetal sheep studies. Using immunohistochemical techniques, we have recently observed that GABAergic neurons of the basal ganglia are affected by repeated umbilical cord occlusion (21). Alterations of GABAergic neurons have been implicated in seizure disor- ders (22). Similarly, injury to the substantia nigra reticulata (GABAergic innervation) precedes seizure induction in adult rats (23). Possibly loss of the striatal GABAergic inhibition is associated with the intermittent cortical seizures in the present study. Alternatively, it is possible that the seizures were initiated in the cortex, causing distal neuronal loss in the striatum by overstimulating the glutamatergic input into the striatum. We have previously shown that prolonged status epilepticus (>30 min) in the cortex can cause distal injury in the hippocampus and lateral cortex (24). However, it is unlikely that the intermittent seizures in the present study caused the striatal neuronal loss observed, because they were of much shorter duration compared with previous studies.

A cumulative effect of repeated insults on postinsult brain edema in adult rats and gerbils after hypoxia-ischemia has been suggested (25). We have previously shown an additive effect of frequently repeated insults on cytotoxic edema during cerebral ischemia in fetal sheep (1). Treatment with the membrane stabilizer ganglioside GM1 improved recovery of primary edema and markedly protected against neuronal loss (26). We did not observe a cumulative effect of repeated insults on cortical cytotoxic edema in the present study. This may be a reflection of where impedance was recorded. Impedance was measured in the parietal cortex region, which was not significantly damaged in the present study. A previous study, measuring striatal tissue impedance after repeated brain ischemia in adult rats, found irreversible changes in impedance after more than two episodes of ischemia, indicating cumulative plasma membrane dysfunction (27).

In summary, we have observed that after recurrent episodes of fetal asphyxia there was a striatal distribution of neuronal loss that is similar to that occurring after repeated cerebral ischemia. Both the progressive hypotension and the increases in the T/QRS ratio that developed with successive insults were related to the degree of neuronal loss. Several postinsult measures, including the duration of recovery of the ECoG intensity and the number of seizures, were associated with the degree of neuronal loss. Therefore, it may be feasible to use these measures clinically to predict outcome. We speculate that recurrent episodes of fetal asphyxia can interact and compromise the cardiovascular response to asphyxia and lead to intrauterine death or a striatal pattern of brain injury in survivors.

\section{REFERENCES}

1. Mallard EC, Williams CE, Gunn AJ, Gunning MI, Gluckman PD 1993 Frequent episodes of brief ischemia sensitize the fetal sheep brain to neuronal loss and induce striatal injury. Pediatr Res 33:61-65 
2. Clapp JF, Peress NS, Wesley M, Mann LI 1988 Brain damage after intermittent partial cord occlusion in the chronically instrumented fetal lamb. Am J Obstet Gynecol 159:504-509

3. Watanabe T, Okamura K, Tanigawara S, Shintaku Y, Akagi K, Endo H, Yajima A 1992 Change in electrocardiogram T-wave amplitude during umbilical cord compression is predictive of fetal condition in sheep. Am J Obstet Gynecol 166:246-255

4. Mallard EC, Gunn AJ, Williams CE, Johnston BM, Gluckman PD 1992 Transient umbilical cord occlusion causes hippocampal damage in the fetal sheep. Am J Obstet Gynecol 167:1423-1430

5. Williams CE, Gunn AJ, Mallard EC, Gluckman PD 1992 Outcome after ischemia in the developing sheep brain: an electroencephalographic and histological study. Ann Neurol 31:14-21

6. Auer RN, Olsson Y, Siesjö BK 1984 Hypoglycemic brain injury in the rat. Correlation of density of brain damage with the EEG isoelectric time: a quantitative study. Diabetes 33:1090-1098

7. Williams CE, Gunn AJ, Gluckman PD 1991 The time course of intracellular edema and epileptiform activity following prenatal cerebral ischemia in sheep. Stroke 22:516-521

8. Gotman J, Gloor P 1980 Automatic recognition and quantification of interictal activity in the human scalp EEG. Electroencephalogr Clin Neurophysiol 41:513-529

9. Gotman J 1982 Automatic recognition of epileptic seizures in the EEG. Electroencephalogr Clin Neurophysiol 54:530-540

10. Gluckman PD, Parsons Y 1983 Stereotaxic method and atlas for the ovine fetal forebrain. J Dev Physiol 5:101-128

11. Vannucci RC, Yager JY 1992 Glucose, lactic acid, and perinatal hypoxic-ischemic brain damage. Pediatr Neurol 8:3-12

12. Gunn AJ, Parer JT, Mallard EC, Williams CE, Gluckman PD 1992 Cerebral histological and electrophysiological changes after asphyxia in fetal sheep. Pediatr Res 31:486-491

13. Rudolph AM 1984 The fetal circulation and its response to stress. J Dev Physiol 6:11-19

14. Jensen A, Berger R 1991 Fetal circulatory responses to oxygen lack. J Dev Physiol $16: 181-207$

15. Papile L-A, Rudolph AM, Heymann MA 1985 Autoregulation of cerebral blood flow in the preterm fetal lamb. Pediatr Res 19:159-161
16. Widmark C, Hokegard KH, Lagercrantz H, Lilja H, Rosen KG 1989 Electrocardiographic waveform changes and catecholamine responses during acute hypoxia in the immature and mature fetal lamb. Am J Obstet Gynecol 160:1245-1250

17. Westgate J, Harris M, Curnow JS, Greene KR 1992 Randomised trial of cardiotocography alone or with ST waveform analysis for intrapartum monitoring. Lancet 340:194-198

18. Rosen KG, Dagbjartsson A, Henriksson BA, Lagercrantz H, Kjellmer I 1984 The relationship between circulating catecholamines and ST waveform in the fetal lamb electrocardiogram during hypoxia. Am J Obstet Gynecol 149:72-77

19. Gall SA, Maier GW, Glower DD, Gaynor JW, Cobb FR, Sabiston DC, Rankin JS 1993 Recovery of myocardial function after repetitive episodes of reversible ischemia. Am J Physiol 33:H1130-H1138

20. Otani H, Engelman RM, Rousou JA, Breyer RH, Lemeshow S, Das DK 1987 The mechanism of myocardial reperfusion injury in neonates. Circulation 76:V161-V167

21. Mallard EC, Waldvogel HJ, Williams CE, Faull RLM, Gluckman PD 1995 Repeated asphyxia causes loss of striatal projection neurons in the fetal sheep. Neuroscience (in press)

22. Houser CR 1991 GABA neurons in seizure disorders: a review of immunocytochemical studies. Neurochem Res 16:295-308

23. Smith M-L, Kalimo H, Warner DS, Siesjö BK 1988 Morphological lesions in the brain preceding the development of postischemic seizures. Acta Neuropathol 76:253264

24. Tan WK, Williams CE, Gunn AJ, Mallard CE, Gluckman PD 1992 Suppression of postischemic epileptiform activity with MK-801 improves neural outcome in fetal sheep. Ann Neurol 32:677-682

25. Nowak TS, Tomida S, Pluta R, Xu S, Kozuka M, Vass K, Wagner HG, Klatzo I 1990 Cumulative effect of repeated ischemia on brain edema in the gerbil. Biochemical and physiological correlates of repeated ischemic insults. Adv Neurol 52:1-9

26. Tan WKM, Williams CE, Mallard CE, Gluckman PD 1994 Monosialoganglioside GM(1) treatment after a hypoxic-ischemic episode reduces the vulnerability of the fetal sheep brain to subsequent injuries. Am J Obstet Gynecol 170:663-670

27. Klein HC, Krop Van Gastel W, Go KG, Korf J 1993 Prediction of specific damage or infarction from the measurement of tissue impedance following repetitive brain ischaemia in the rat. Neuropathol Appl Neurobiol 19:57-65 